\title{
Cloud computing service center model for medical service and data processing
}

\author{
Mo ZhiYi, Xu Ch uanBen, Li Qing \\ Wuzhou University, Wuzhou, 543002, China \\ Email: mofly_0214@qq.com
}

Key words: Clouding Computing, Technology, Medical information, Storage

\begin{abstract}
When entering a new stage of socialism, science and technology have developed rapidly. The technology represented by computer and internet has been used widely in all walks of life. Cloud computing technologies and concepts, as a new invention, not only change the original mode of computing resources, but also simplify the work flow and improve the internal management, which has made a contribution to improve work efficiency. As is known to all, the medical work has always been a focus of the country and the people, and the hospital management is diversity of the content, complicated and overloaded and important. It will be a new situation for the hospital development to achieve the cloud computing and management in medical construction by using the information technology. Cloud computing technologies and concepts shall be stated in this paper, and the application shall be achieved to promote the development of medical and health services of the whole society, combining with the information construction of medical.
\end{abstract}

\section{Introduction}

Nowadays, the economy of our country develops rapidly, and modern technology also presents a new reform under the drive of economy. With the continuous improvement of people's life quality, healthcare has been more and more attention. In a sense, the development of medical and health services can not be separated from powerful technical support, connecting with information construction and promotion, which contributes to provide more convenient service for common people to quicken the sharing and application of information. Clouding computing is a theory of large data, which can achieve focus utilization of various resources by automated management and conservation of information and data, and of which function is self-evident in the healthcare reform. Based on the status of presently medical treatment of hospital, we must set about the popularization and application of computing ideas and technology to establish perfect service system of clouding computing ideas and complete practically propulsion, which can lay a solid foundation for the development of medical and health services.

\section{Related overview of clouding computing}

\section{(I) Concept and Theory}

The use basis of clouding computing is the disclosure of service and standard, which puts the network system in the central location, and then considers the confidentiality of user information from multi-angle, and conveniently disposes the data to achieve the service of network operation in the end. In other words, a great deal of work in the model of clouding computing is not the information stored in personal computers and phones, but the contents stored in server by internet operation. The enterprises only need to be responsible for the cloud services and computing management, which can ensure the high run of system to develop the powerful function of clouding computing and provide enough capacity for customer use.

(II)Development prospect of clouding computing.

With the continuous improvement of information and intelligent technology, high technology industry has developed rapidly. As a new technology measure, clouding computing is not only the pronoun of high tech, but also has great commercial value, closely related to daily life of people. 
Nowadays, many companies have achieved the convenient management by the application of clouding computing, reduced the investment of human resources and established corresponding software services to provide multiple choices for the supplier. At the same time, the future development of clouding computing shall be bound to be more rapid and shall be widely used in many fields, such as online game, pay TV and other personalized services, thus bringing great influences for life.

\section{Relationship between large data and clouding computing}

In recent years, Cloud computing is popular with academia and industry. Then large data turns out and more popular, which shall be frequently bracketed with clouding computing by someone. So, what is the relationship between the large data and clouding computing? Large data and clouding computing have something in common in reality, which are served for the data storage and process and both of them need to take up a lot of storage and computing resources, and the massive data storage technology, mass data management technology, MapReduce and other parallel processing technology used in large data are also the key technology of cloud computing. However, both of them are different in many ways. The purpose of clouding computing is to transfer preferably, expand and manage computing and storage resources and ability through the internet to save the IT deployment cost of the enterprise, of which processing object is IT resources, processing capacity and various application. Clouding computing changed the IT frame of the enterprise from the root. The main driving forces of industrial development are the manufactures of storage and computing equipment and the companies with the computing and storage resources. But, the purpose of large data is to digest information in mass data to find out the value of data, of which processing object is various data. Large data makes the enterprise from the "business-driven" into "data-driven", thus changing the business structure of the enterprise, of which direct beneficiaries are not the IT department but the operating department or CEO of the enterprise. The main driving forces of industrial development are the software companies occupied in the storage and process of data and the companies with mass data. Therefore, it is easy to find the relationship between clouding computing and large data which is the tools and application in reality, in other words, on the one hand, the clouding computing provides powerful tools and ways for large data, and on the other hand, the large data provides valuable place for clouding computing. large data can be interpreted as the extension of clouding computing from the use of technology. The combination of large data and clouding computing shall bring out the best in each other and can play the greatest advantage. Clouding computing can provide powerful storage and computing for large data to process the abundant information of mass data more quickly and provide more conveniently services. However, the business requirements from the large data shall provide more and better practical application for the landing of clouding computing. Certainly, the appearance of large data shall make clouding computing face new challenges.

\section{Medical informatization based on clouding computing ideas and technology}

The practical application of service for medical informatization in our country has a lot of disadvantages because the clouding computing ideas and technology are not widely used in it and the later start. At present, the classification on application of clouding computing ideas related to it in the medical unit is not clear enough and the developed construction standards are varied. The differences in investment are obvious due to the influence of balance for medical condition and regional development, and the upgrading of hardware and software is difficult. At the same time, the communication between departments of the hospital is not smooth enough, which go against the popularities of clouding computing. Aimed at these problems, medical industry must improve the clouding computing ideas and complete the popularization and application of technology.

(I) Software services

Making use of the software services provided by service provider of clouding computing, local hospitals can share the infrastructure provided by the system and develop their own characteristic 
software in line with the requirements of self-development, which greatly reduce the cost paid by hospital for software at present, and reduce a series of problems of irregularity and non-uniform technical standards for the development of information system in all hospitals.

(II) Hardware lease

The hospitals have a great demand for the purchase of server and also have a great of cost on the management and maintenance in the development of the information construction by themselves. Some small and medium hospitals can not follow the step of information construction because of the relative shortage funds, which can not have substantial development for this reason. Particularly in the area of slow development, provide the guarantee for their medical information construction, and further reduce the gap with areas that are fairly developed economically. The statues of healthcare informatization which are unpopular and non-uniform in the construction is greatly improved.

(III) Data storage

The greatest advantage of clouding computing ideas and technology is that the mass data can be integrated intensively to provide a shared platform of resources and information for medical institutions. Maximum ensure the fair use of information by clouding computing, complete the communication and transfer of resources and get rid of construction island to promote the modernization construction and development of hospital.

(IV) Data analysis

Although the relevant medical data of hospital is only the original collection and arrangement at the present stage, the demand of the practitioners shall not stop there. On this account, the analysis and calculation about data provided by clouding computing can process refinedly the mass medical data and effective use. The improvement of the medical quality shall make the diagnosis of practitioners more effective and the quality of diagnosis more superior, and also further ensures the health of patients and greatly provides the welfare for people.

Large data also has risk, one of which is the disclosure of user's privacy and the security risk of data. The data privacy and privacy problem shall be greater problem in future years due to the production, storage and analysis of mass data, which makes the enterprise begin to study the mew protective measure of data as soon as possible. However, telecom operators have advantages in the network security and data center security and other way, such as on this basis, the establishment of advantages for the security assurance in the entire large data field shall make benefit the development of large data. The arrival of the cloud computing and large data era makes the whole society increasingly become a whole, and in such system, protection of personal privacy has become the important basis of the construction for the social credit system. While encouraging innovation and progress, we must be acutely aware of that it is choicely and purposefully for the use and open of the clouding computing and large data, no matter the USA or any country, but which is not openly without principle, which is not only limited by laws and regulations, but also is closely related to integral development plan and global strategy for a whole country. The efforts made in the protection of personal privacy, are not only the protection for all members of society but also the protection for the state security and long-term sustained and healthy development of society.

\section{Conclusion}

In conclusion, information technology has irreplaceable function in modernization development and enterprise construction with the arrival and development of information era. Medical industry as the patron saint of people will certainly evolve toward to intelligence and technology in the new background. Clouding computing ideas and technology can achieve the rational use of resources, ensure the sharing and transfer of information and bring about the greatest impact on reform for internet IT industry on the basis of efficient, flexible and low-cost advantages. The hospital must grasp the information advantage, optimum the construction structure, play greatest connotation power and dig the potencies to complete the information construction system of hospital in medical. 


\section{Acknowledgement}

General projects of science and technology research in Guangxi universities in 2014(YB2014358), Guangxi Colleges and Universities Key Laboratory of Professional Software Technology(Wuzhou University).

\section{Reference}

[1] Gupta P, Seetharaman A, Raj J R. The usage and adoption of cloud computing by small and medium businesses[J]. International Journal of Information Management, 2013, 33(5):861-874.

[2] Espadas J, Molina A, Jiménez G, et al. A tenant-based resource allocation model for scaling Software-as-a-Service applications over cloud computing infrastructures[J]. Future Generation Computer Systems, 2013, 29(1):273-286.

[3] Andrikopoulos V, Binz T, Leymann F, et al. How to adapt applications for the Cloud environment[J]. Computing, 2013, 95(6):493-535.

[4] Zheng Y L, Ding X R, Poon C C, et al. Unobtrusive sensing and wearable devices for health informatics.[J]. IEEE transactions on bio-medical engineering, 2014, 61(5):1538-54.

[5] Morenovozmediano R, Montero R S, Llorente I M. Key Challenges in Cloud Computing: Enabling the Future Internet of Services[J]. IEEE Internet Computing, 2013, 17(4):18-25. 